\title{
Assemblages non soudés dans les réacteurs nucléaires
}

\author{
Jean Dhers ${ }^{*}$ \\ AREVA NP, 10 rue Juliette Récamier, 69006 Lyon, France
}

Reçu le 17 octobre 2017 / Accepté le 13 décembre 2017

\begin{abstract}
Résumé. Les réacteurs nucléaires utilisent peu d'assemblages non soudés. Il existe cependant quelques assemblages sans soudure qui concernent le circuit primaire des réacteurs LWR, tels que ceux de nos centrales françaises. Il s'agit essentiellement de trois types d'assemblage : le dudgeonnage, le frettage et le boulonnage. Les deux premières techniques concernent respectivement le générateur de vapeur, les pénétrations dans le couvercle, ou le fond de cuve et la pompe primaire. Elles sont toujours associées à un soudage ultérieur qui garantit l'étanchéité. Il s'agit de composants essentiels au bon fonctionnement du réacteur. Les assemblages boulonnés concernent la pompe primaire, l'assemblage du couvercle de cuve avec la cuve, et les internes de cuve. Certains assemblages boulonnés sont plus sensibles aux phénomènes de corrosion sous tension voire pour les internes de cuve de corrosion sous tension assistée par irradiation.
\end{abstract}

Mots clés : assemblages / nucléaire

\begin{abstract}
Non welded assemblies in nuclear reactors. Few non welded assemblies are present in nuclear reactors. There are however some weldless assemblies which concern the Light Water Reactors. It is principally about three assembly types: expansion, shrink-fitting, and bolting. The two first technics concern respectively steam generator, penetrations in the closure head of the reactor pressure vessel, the vessel bottom head and the reactor coolant pump. They are always associated with a subsequent welding which warrants tightness. It is about essential components in the smooth running of the reactor. The bolted assemblies concern the reactor coolant pump, the assembly of the reactor vessel closure head with the vessel, and reactor internals. Some bolted assemblies are more sensitive to stress corrosion phenomena, or even for the reactor internals, to stress corrosion assisted by irradiation.
\end{abstract}

Keywords: assembly / nuclear

\section{Introduction}

Un réacteur nucléaire est un assemblage de diverses pièces métalliques forgées, moulées ou laminées. La technique principale d'assemblage est le soudage, afin de garder une étanchéité totale et aussi pour conserver une résistance mécanique suffisante.

Les circuits primaires des réacteurs à eau sous pression (comme nos réacteurs français) présentent cependant quelques assemblages non soudés. À noter cependant que si ces assemblages doivent présenter aussi une fonction d'étanchéité on devra ajouter une soudure d'étanchéité (exemples des soudures des tubes sur la plaque tubulure ou des adaptateurs de commande de grappe sur le couvercle de cuve).

Les assemblages retenus dans cette présentation concernent le frettage de tubes (pénétration de tubes dans

\footnotetext{
* e-mail: jean.dhers@areva.com
}

le couvercle de cuve ou le fond de cuve, le rouet de pompe sur l'axe de la pompe), l'expansion hydraulique ou le dudgeonnage pour les tubes du générateur de vapeur, et enfin le boulonnage (divers éléments boulonnés dans la pompe primaire, et boulonnage du couvercle de cuve sur la cuve, internes de cuves).

\section{Frettage}

Les assemblages par frettage concernent essentiellement trois types de composants :

- les pénétrations de tubes dans le couvercle de cuve pour laisser passer les barres de contrôle;

- les pénétrations de tubes avec le fond de cuve pour laisser passer l'instrumentation;

- le rouet de pompe sur l'axe de la pompe.

L'assemblage des tubes de pénétration avec le couvercle ou le fond de cuve devant avoir aussi une fonction d'étanchéité, elles sont complétées par un soudage TIG. 


\subsection{Pénétrations de tube dans la cuve}

\subsubsection{Assemblage adaptateur/couvercle de cuve}

Le tube en inconel 690 (épaisseur $16 \mathrm{~mm}$ ) permet de passage des lignes de commande de grappe. Le couvercle de cuve est en acier 16MND5 revêtu d'acier inoxydable austénitique en 309 et 308L (Fig. 1).

L'assemblage s'effectue par frettage thermique, le tube est refroidi dans l'azote liquide est introduit dans le trou du couvercle de cuve.

L'étanchéité tube-cuve est réalisée par le soudage TIG de fil en 690.

\subsubsection{Problèmes rencontrés}

Une perte d'étanchéité a été observée aussi bien en France [1], qu'aux États-Unis [2] au niveau de l'assemblage. Cette perte a entraîné des fuites et a conduit au remplacement du couvercle de cuve.

Des fissures intergranulaires ont été observées sur la paroi interne des tubes.

La cause de cette fissuration est la conjonction d'une contrainte résiduelle de soudage liée avec un milieu corrosif (acide borique). Le matériau utilisé à cette époque pour le tube et la soudure était l'inconel 600 moins chargé en Cr. Le remplacement systématique de l'inconel 600 par le 690 (plus chargé en $\mathrm{Cr}$ ) [3] pour les tubes et la soudure a permis d'améliorer la tenue de l'assemblage.

\subsubsection{Assemblage tubes pour instrumentations/fond de cuve}

Le tube en inconel 690 permet le passage d'instrumentation du cœur. Le fond de cuve est en acier 16MND5 revêtu d'acier inoxydable austénitique en 309L et 308L.

L'assemblage s'effectue par frettage dans les mêmes conditions que pour les adaptateurs.

Les problèmes apparus sont de même nature à savoir fissuration au niveau de la soudure en J [4].

La conception du réacteur EPR a pris en compte les risques liés à ces assemblages de fond de cuve, et les a supprimés.

\subsection{Assemblage roue/arbre}

La roue de pompe primaire est un élément fondamental du fonctionnement et de la sûreté des réacteurs à eau pressurisée. En effet, elle assure la circulation de l'eau du circuit primaire du générateur de vapeur vers le réacteur (Fig. 2).

La roue pour les réacteurs français est en acier inoxydable austénoferritique moulé Z3CN20-09 (CF3).

L'axe est en acier forgé Z6CNNb18-11 (347).

Dans le cas des réacteurs français, l'assemblage de la roue de pompe avec l'arbre qui est entraîné par le moteur se fait par frettage.

La roue est chauffée à une température de $200^{\circ} \mathrm{C}$ environ et emmanchée dans l'arbre.

Le principal souci qui a été (rarement) rencontré est le défrettage de la roue.

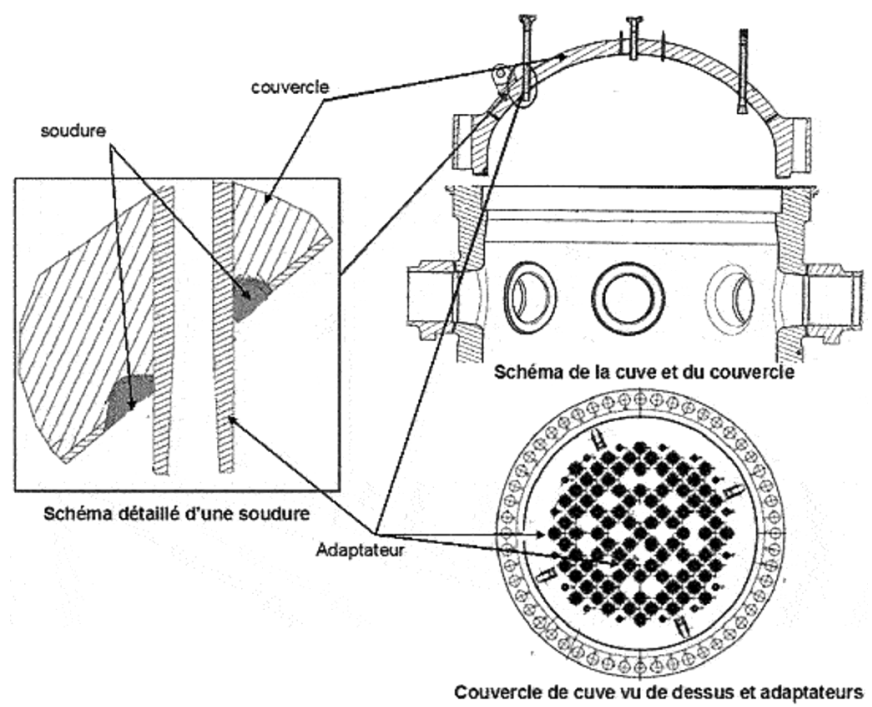

Fig. 1. Assemblage adaptateur/ couvercle de cuve.

Fig 1. Reactor pressure vessel head penetration.

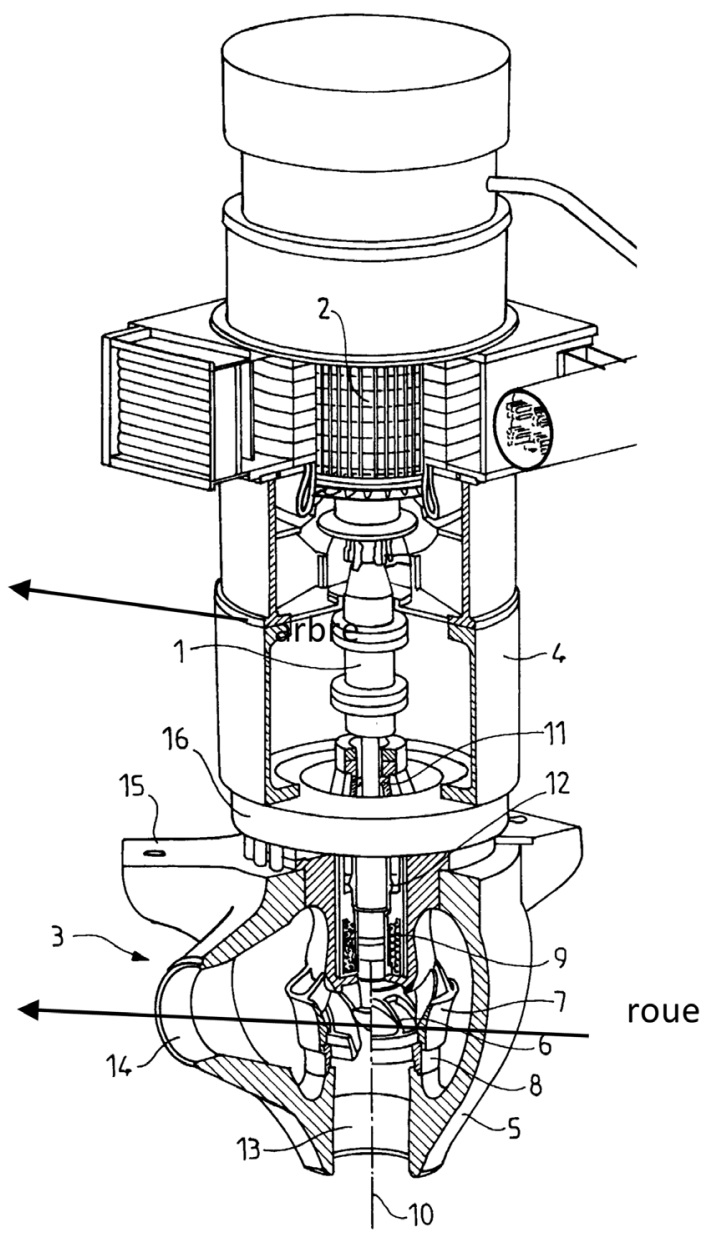

Fig. 2. Pompe primaire.

Fig 2. Primary pump. 


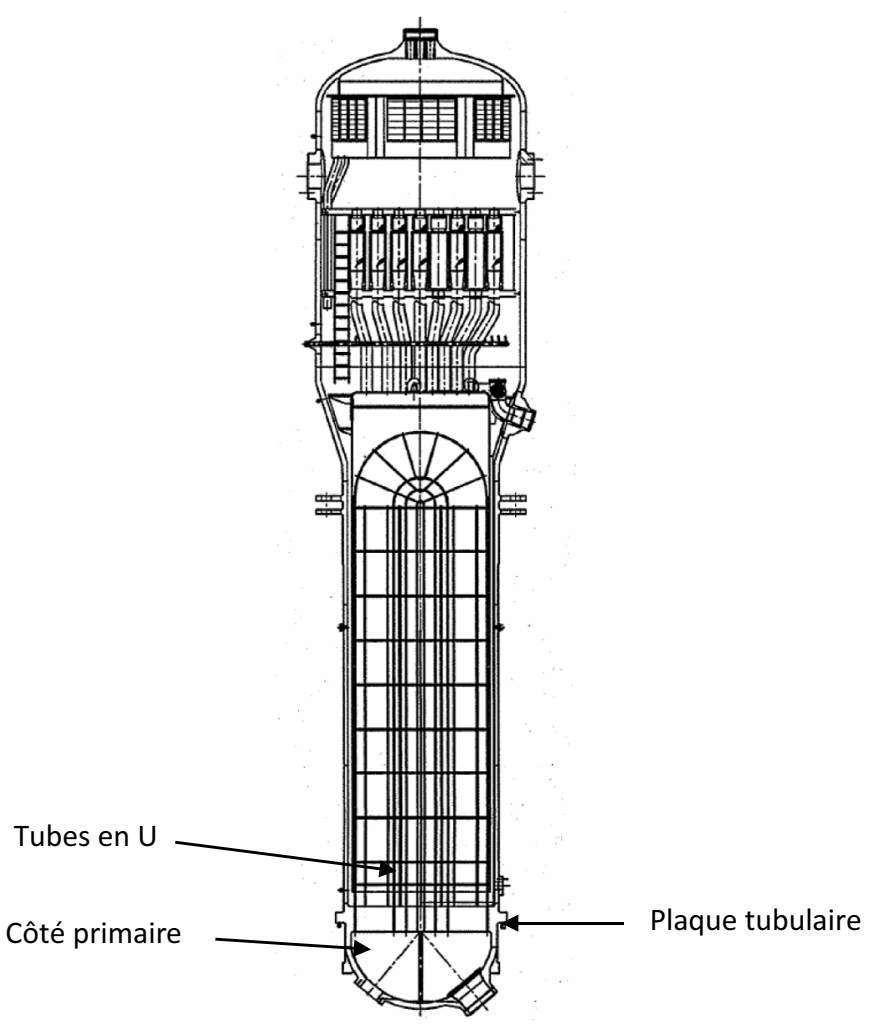

Fig. 3. Générateur de vapeur.

Fig 3. Steam generator.

Les causes du défrettage potentiel sont:

- la dilatation thermique différentielle;

- le surfrettage en arrêt à chaud et plastification du moyeu ;

- les transitoires thermiques au démarrage.

Pour les pompes 100 (CP1300, Chine, Corée) et 93D (CP900, Koeberg), la roue est frettée sur une portée conique de l'arbre. Un écrou bloque la roue axialement sur l'arbre. Une clavette permet de transmettre le couple en cas de défrettage de la roue. Si la roue se défrette, l'écrou maintient la roue sur l'arbre et la clavette continue à transmettre le couple

Une amélioration a été réalisée sur les pompes 100 et appliquée aussi sur les pompes de nouvelles générations. Elle consiste en un arbre creux, et un écrou avec pompe auxiliaire qui permet de réchauffer l'arbre lors des transitoires d'arrêt et démarrage de la pompe à chaud, et ainsi réduire les risques de défrettage.

\section{Dudgeonnage et expansion hydraulique}

Les assemblages par dudgeonnage et expansion hydraulique concernent uniquement l'assemblage des tubes de générateur de vapeur avec la plaque tubulaire (voir Fig. 3).

Les tubes sont en inconel 690, la plaque tubulaire est en acier 18MND5 revêtu d'inconel 690 du côté circuit primaire uniquement.

Une plaque tubulaire de réacteur EPR contient environ 10000 trous usinés par perçage profond.

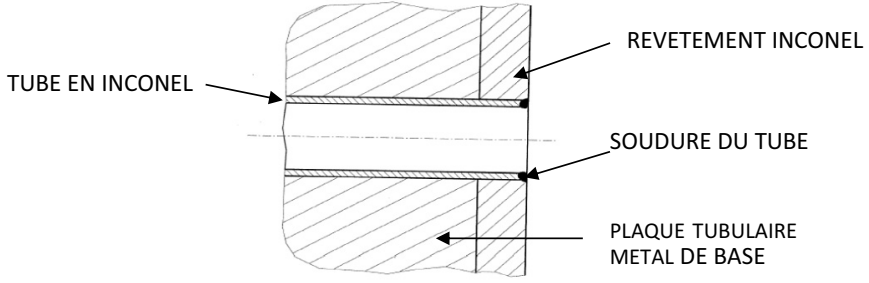

Fig. 4. Dugeonnage d'accostage et soudure d'un tube sur la plaque tubulaire.

Fig 4. Dugeonnage of fit-up and weld of a tube on the tubesheet.

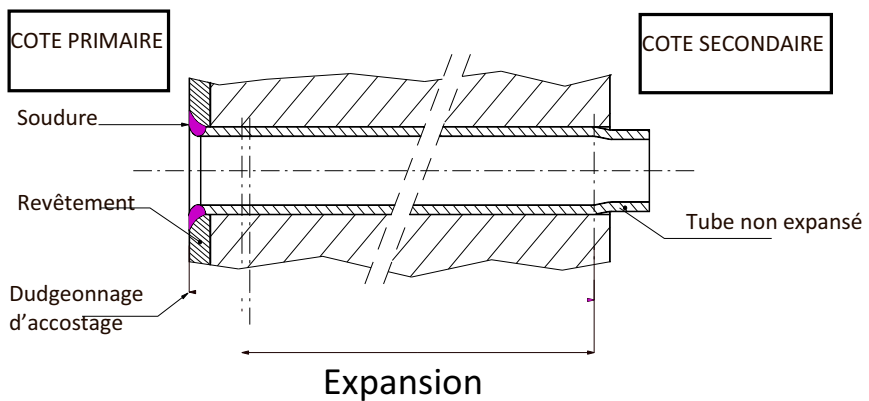

Fig. 5. Dudgeonnage côté primaire et expansion hydraulique côté secondaire.

Fig 5. Tube dugeonnage primary side and expansion on the secondary side.

\subsection{Dudgeonnage d'accostage}

Le dudgeonnage d'accostage consiste à venir plaquer par expansion mécanique sur les parois de l'alésage de la plaque tubulaire le tube d'échange (voir Fig. 4) en inconel 690. Il se situe du côté circuit primaire, donc au niveau de la plaque tubulaire en acier 18MND5 revêtue inconel.

Il est suivi d'une opération de soudage d'étanchéité (soudage TIG sans métal d'apport).

Il est réalisé à l'aide de galets qui vont par déformation plastique plaquer le tube contre l'alésage.

Une corrosion aux joints de grains a affecté de nombreux tubes en Alliage 600 côté primaire dans des zones à fortes contraintes résiduelles des extrémités dudgeonnées.

L'Alliage 690, dont la teneur en chrome est le double, a remplacé l'Alliage 600 dans les nouveaux GV. Des améliorations dans la mise en œuvre (accostage du tube sur la plaque par mandrin hydraulique, traitement de détensionnement, etc.) ont été aussi apportées.

\subsection{Expansion hydraulique}

L'assemblage dudgeonné du tube sur la plaque tubulaire laissait subsister un interstice côté secondaire qui a été à l'origine de phénomènes de fissuration de tubes. L'expansion hydraulique côté secondaire de la plaque permet de prévenir les infiltrations d'eau et par là la corrosion en pied de tube (Fig. 5).

L'expansion hydraulique est réalisée par expansion du tube sous une haute pression d'eau. 


\section{Assemblages boulonnés}

Les assemblages boulonnés concernent plusieurs composants du réacteur : le couvercle de cuve, les internes de cuve tels que le réflecteur lourd, et la pompe primaire.

\subsection{Couvercle de cuve}

La fermeture par boulonnage de la cuve du réacteur doit assurer l'étanchéité totale de la cuve (Fig. 6 et 7).

La procédure de fermeture prévoit:

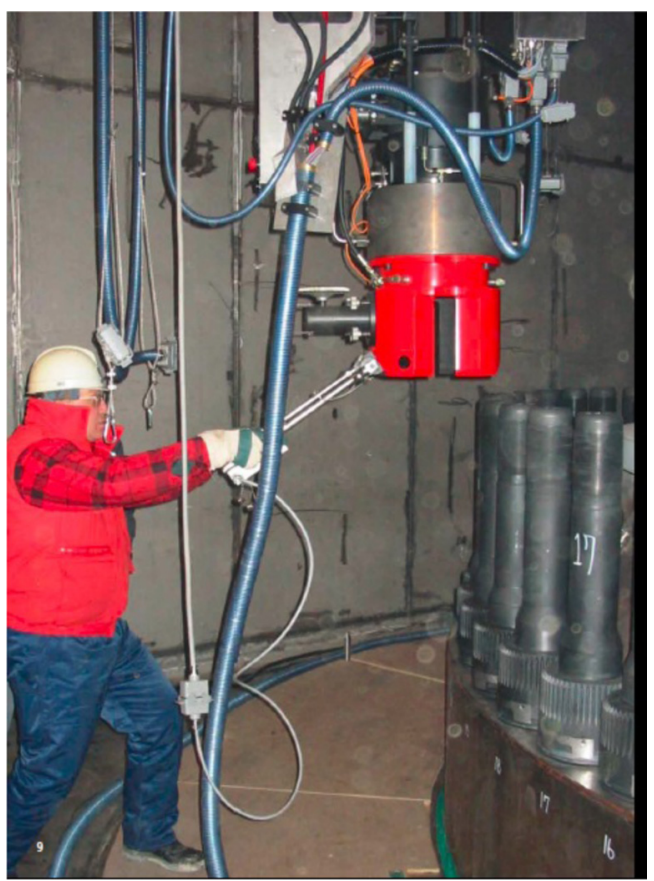

Fig. 6. Dévissage des goujons de cuve.

Fig 6. Unscrewing of reactor pressure vessel studs.
- la pose du couvercle sur la cuve du réacteur ;

- la pose de la machine de serrage et desserrage des goujons de cuve sur le couvercle;

- le vissage et serrage des goujons de cuve.

Les problèmes majeurs sont dus ici à la qualité du taraudage des trous sur la bride de cuve.

\subsection{Visserie dans les pompes primaires}

L'assemblage des différents composants d'une pompe primaire de réacteur utilise différentes technologies dont la boulonnerie/visserie (voir Fig. 8).

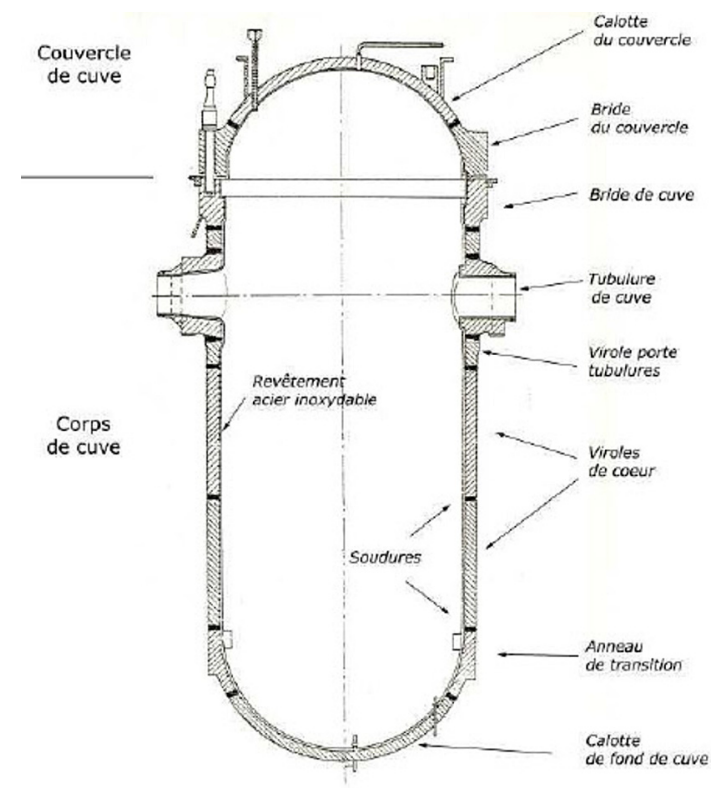

Cuve de réacteur 1300 MWe

Fig. 7. Cuve de réacteur.

Fig 7. Reactor pressure vessel.

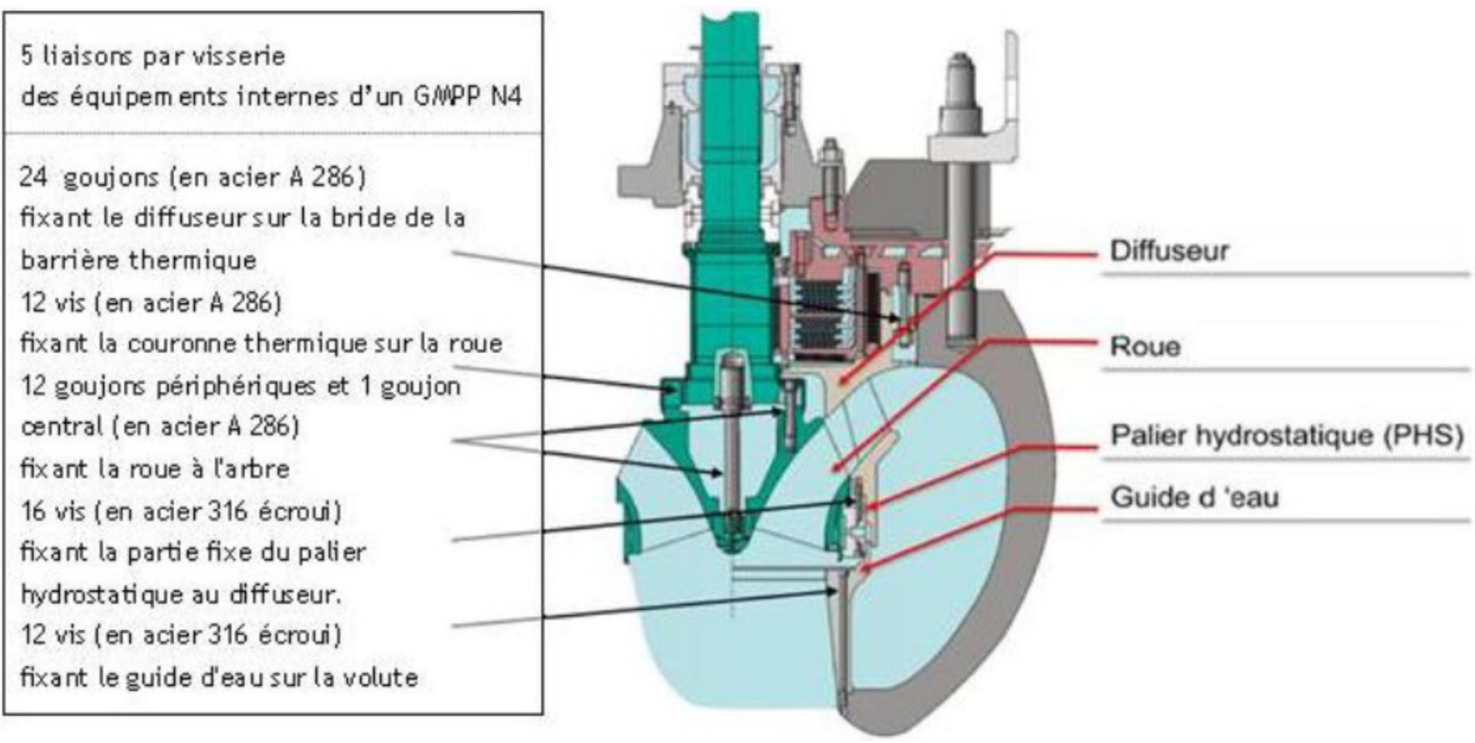

Fig. 8. Liaison par visserie des équipements internes d'une pompe de réacteur $1450 \mathrm{MW}$ [5].

Fig 8. Connections by screws of internal equipments of a primary pump of $1450 \mathrm{MW}$ a nuclear reactor [5]. 


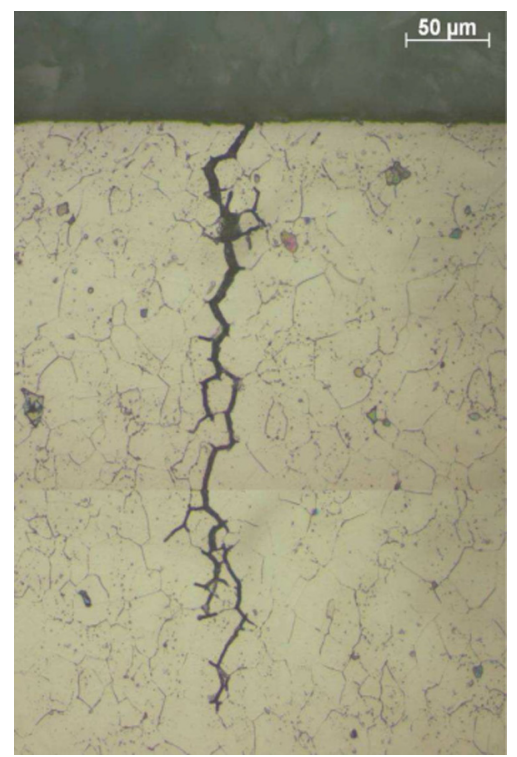

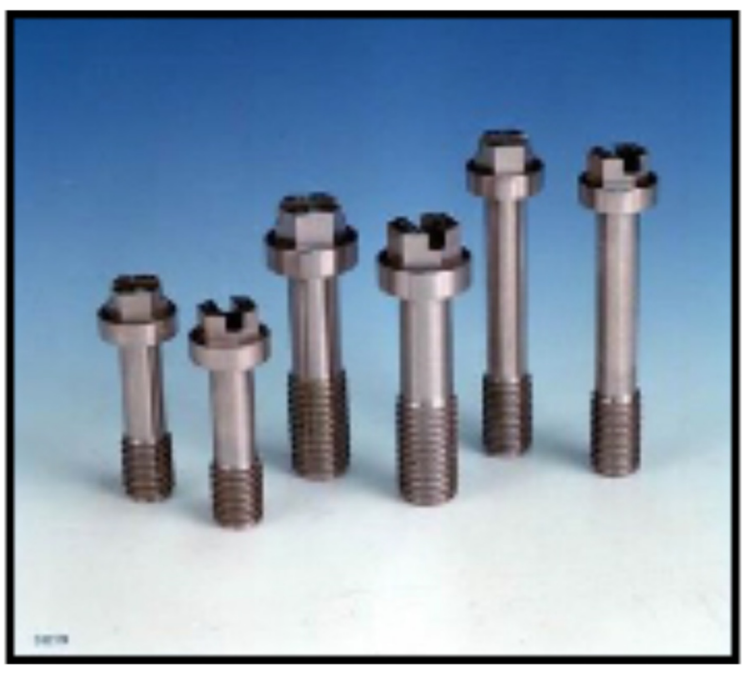

Fig. 11. Baffle bolts.

Fig. 9. Fissure dans un goujon en acier A286.

Fig 9. Crack in a A286 steel stud.

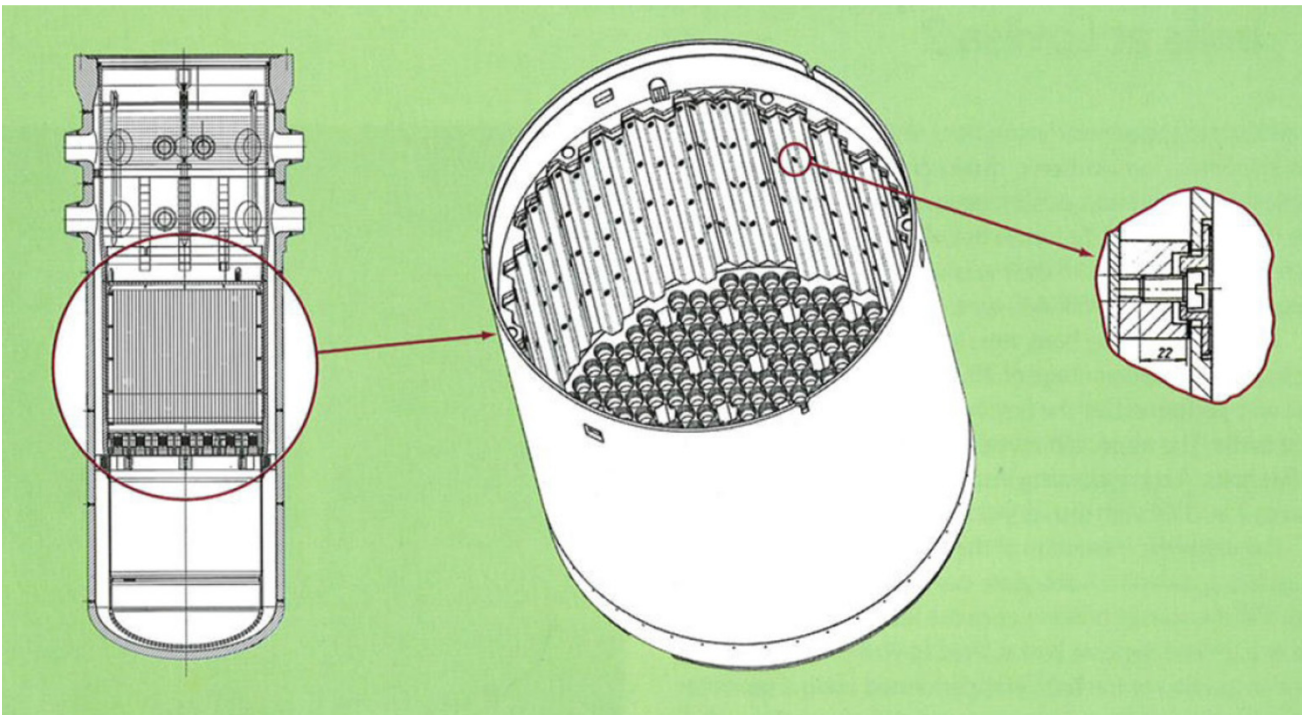

Fig. 10. Interne de cuve et baffle bolt.

Fig 10. Internal pressure vessel and baffle bolt.

Deux nuances d'acier inoxydable sont utilisées, le 316L écroui et l'A286 (X5NiCrTi 26-15).

Des fissures de corrosion sous contraintes ont été observés sur certains goujons et vis [5], essentiellement en A-286 (Fig. 9). Cette nuance sera abandonnée dans la mesure du possible dans l'avenir.

Les autres voies d'améliorations sont la modification de la géomètrie des goujons et boulons, et la limitation de la dureté en surface des goujons, par amélioration de la fabrication.

\subsection{Baffle bolts}

Les baffles bolts en acier inoxydable austénitique (347, 316, 304) sont des boulons qui tiennent la structure d'interne du réacteur (voir Fig. 10 et 11) qui permet d'orienter le flux d'eau de refroidissement et de positionner le cœur.

Ces boulons sont soumis à des contraintes de par leur fonction, de serrage, et sont dans un environnement d'eau du circuit primaire. La fabrication des boulons (usinage, roulage des filets) peut induire un durcissement et des contraintes résiduelles de traction.

Des fissurations dues à de la corrosion sous contrainte sont apparues dans différents réacteurs français, américains et russes [6-8]. Dans certains cas l'irradiation peut être un facteur aggravant, les internes se trouvant soumis à une dose élevée (proximité avec le cœur).

Les solutions apportées pour prévenir ces endommagements consistent à changer la matière (passer du 347 à l'acier 316L écroui), modifier la géométrie des goujons pour 


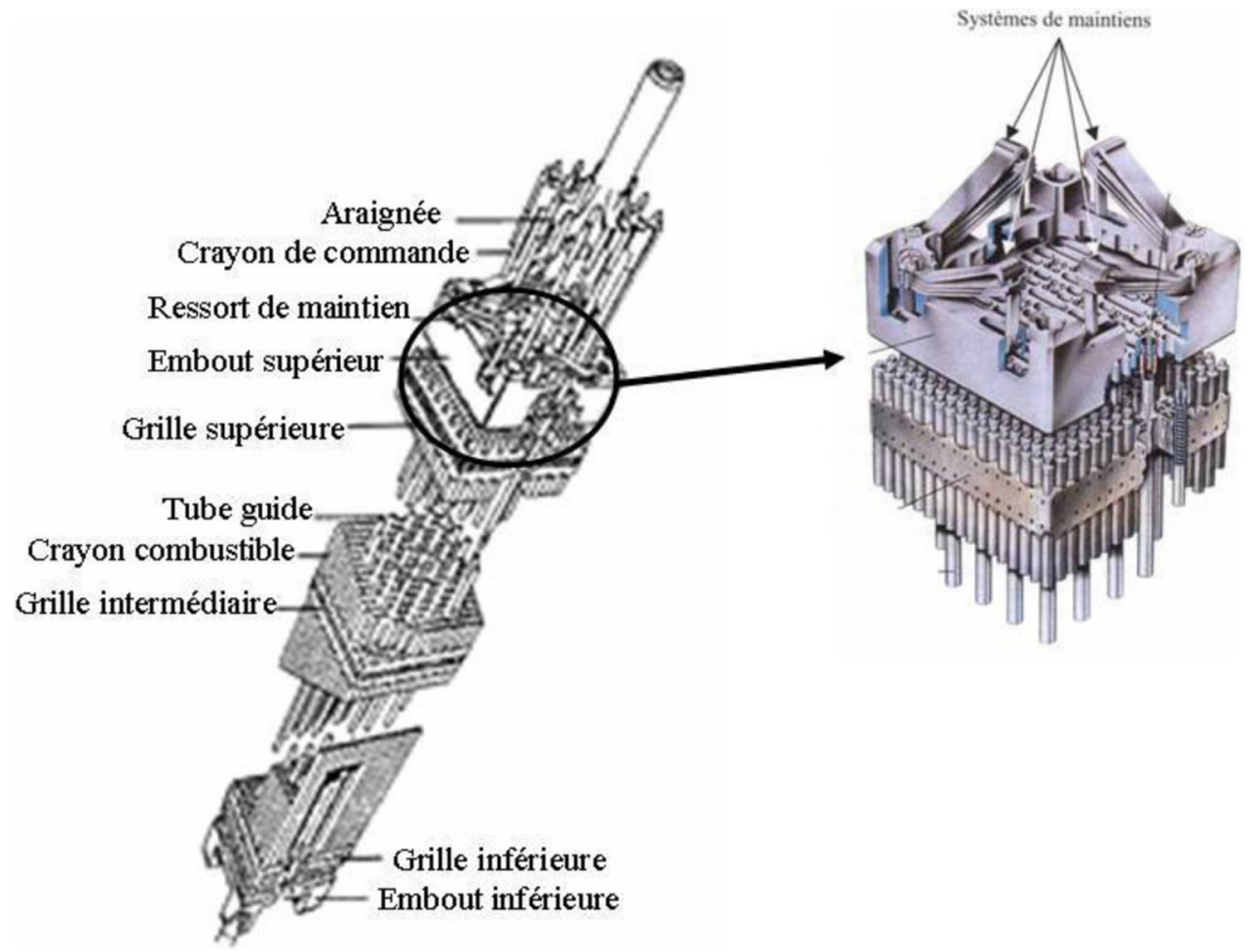

Fig. 12. Assemblage combustible.

Fig 12. Fuel assembly.

diminuer les effets de concentration de contrainte, et adapter les techniques de fabrication pour améliorer la surface.

\section{Divers autres assemblages}

Parmi les autres assemblages non soudés des centrales nucléaires deux sont ici pris en compte:

- le maintien des crayons de combustibles par des ressorts plats en inconel 718 (Fig. 12). Le ressort soumis à des conditions sévères doit résister le temps de vie de l'assemblage soit moins de cinq ans environ. Le risque majeur est la corrosion sous tension du ressort. Pour prévenir ce dommage le traitement thermique du ressort est optimisé ;

- le rivetage, très utilisé dans l'aéronautique, était ignoré dans le nucléaire. Le réacteur de recherche Jules Horowitz (RJH) a introduit cette technologie d'assemblage dans le nucléaire. Le procédé de rivetage a été retenu pour l'assemblage de tôles en alliage d'aluminium 6061-T6 du bloc réflecteur du réacteur RJH. Les rivets sont également en 6061-T6. Le rivetage est un mode de fabrication qui n'est pas couvert par le RCC-MX 2008.

\section{Références}

1. J. Économou, et al., Contrôle et expertise métallurgique de traversée de couvercle de cuve, Fontevraud III, 1994

2. NRC, Bulletin: 2001-01: Circumferential Cracking of Reactor Pressure Vessel Head Penetration Nozzles, 2001

3. B. Normand, Prévention et lutte contre la corrosion: une approche scientifique et technique, 2004

4. NRC Inspection Manual, Temporary instruction 2515/152, Rev. 1 reactor Pressure vessel Lower penetration Nozzles, NRC Bulletin 2003-02, 2003

5. Le point de vue de l'IRSN sur la sûreté et la radioprotection du parc électronucléaire français en 2012. Rapport IRSN/DG/ 2013-00005

6. J.J. Regidor, et al., Inspection and replacement of baffle former bolts in VVER-440 reactor type, 6th International Conference on NDE in Relation to Structural Integrity for Nuclear and Pressurized Components October 2007, Budapest, Hungary

7. Westinghouse NSAL-16-1, Revision 1, "Baffle-FormerBolts", August 9, 2016

8. Information Notice No. 98-11: Cracking of Reactor Vessel Internal Baffle Former Bolts in Foreign Plants. United States Nuclear Regulatory Commission Office of Nuclear Reactor Regulation Washington, D.C. 20555-0001 March 25, 1998 